\title{
Análisis cualitativo de la gestión tecnológica para la innovación de servicios financieros: Estudio de casos múltiples de startups FinTech en Lima Metropolitana'
}

\author{
Héctor Guardamino', Marta Tostes ${ }^{2}$
}

\author{
${ }^{1}$ Facultad de Gestión y Alta Dirección, Pontificia Universidad Católica del Perú, Perú | \\ hector.guardamino@pucp.pe | https://orcid.org/0000-0000-0000-0000 \\ ${ }^{2}$ Departamento Académico de Ciencias de la Gestión, Pontificia Universidad Católica del \\ Perú, Perú | mtostes@pucp.edu.pe | https://orcid.org/0000-0003-0855-7463
}

\begin{abstract}
Resumen: El presente estudio de casos múltiples analiza, desde un enfoque cualitativo, la gestión de la tecnología para la innovación de servicios financieros en cuatro startups FinTech de Lima Metropolitana en Perú: Apurata, Difondy, TasaTop y Tranzfer.me. Para evaluar los procesos de gestión tecnológica de estas FinTech, se utilizó el modelo de las Seis Facetas (Kearns, Taylor \& Hull, 2005). Además, se diseñó una rúbrica a partir de criterios y subcriterios. Posteriormente, como técnica de recolección de información, se aplicaron entrevistas a profundidad en torno a cada uno de los seis procesos del modelo (variables) y sus respectivos principios (subvariables). Por otro lado, para ordenar y sistematizar la información recolectada se utilizó el software de gestión de contenido WebQDA. Finalmente, los resultados muestran que el modelo de las Seis Facetas es una potencial herramienta para analizar los procesos de gestión tecnológica que se gestan en las FinTech con un área de tecnología interna. Esto con el fin de encontrar puntos de mejora cuya solución contribuya a la innovación de servicios de las FinTech, impactando en su competitividad. Asimismo, entre las FinTech estudiadas, el proceso tecnológico más sólido es el planeamiento, mientras que el proceso en el que más adolecen es el de formación de clientes. Por último, se concluyó que TasaTop es la FinTech más preparada para la innovación de sus servicios.
\end{abstract}

Palabras clave: Análisis Cualitativo; FinTech; Gestión de la Tecnología; Innovación de Servicios Financieros.

Qualitative Analysis of the Technological Management for the Innovation of the Financial Services: Multiple Cases Study of Startups FinTech in Metropolitan Lima

\begin{abstract}
This multiple-case study analyses, from a qualitative approach, the technological management for the innovation of the financial services in four startups FinTech from Metropolitan Lima in Peru: Apurata, Difondy, TasaTop and Tranzfer.me. The Six Facets mode (Kearns et al., 2005) was used to assess the technological management processes of these FinTech. In addition, a rubric was developed from criteria and sub-criteria. Subsequently, thoroughly interviews were applied as a technique to collect information related to each of the six model processes (variables) and their respective principles (subvariables). On the other hand, the collected information was organized and systematized using the data content management software WebQDA. Finally, the results indicate that the Six Facets model is a potential tool to analyze the technological management processes that are developed in FinTech with internal technology areas. This in order to be able to find points of improvement whose solution contributes to the innovation of FinTech services, impacting its competitiveness. Likewise, among the studied FinTech, the most solid technological process is the planning, whereas the process in which the analyzed FinTech suffer the most is in the process of customer formation. Finally, it was determined that TasaTop is the most prepared FinTech for the innovation of its services.
\end{abstract}

Keywords: Qualitative Analysis; FinTech; Technology Management; Financial Services Innovation.

\footnotetext{
${ }^{1}$ Esta investigación se sustenta de forma amplia en la Tesis de Licenciatura titulada "Gestión tecnológica para la innovación de servicios financieros: Estudio de casos múltiples de startups FinTech en Lima Metropolitana", FGAD, PUCP, 2020: http://tesis.pucp.edu.pe/repositorio/handle/20.500.12404/18306
} 


\section{Introducción}

La evaluación de los procesos de gestión tecnológica en empresas inmersas en sectores de alta turbulencia tecnológica que cuentan con un área de tecnología interna, es adecuada a partir de un enfoque cualitativo (Liao, Hull \& Sriramachandramurthy, 2013). En este sentido, el objetivo de esta investigación se orienta a analizar los procesos de gestión tecnológica para la innovación de servicios financieros en cuatro startups FinTech de Lima Metropolitana en Perú: Apurata (FinTech de préstamos), Difondy (FinTech de crowdfunding), TasaTop (FinTech Marketplace de ahorro e inversión) y Tranzfer.me (FinTech de transferencias internacionales), a partir del modelo de las Seis Facetas de Kearns et al. (2005). De igual modo, con este objetivo, se busca responder a la pregunta acerca de cómo gestionan sus procesos de tecnología estas FinTech seleccionadas. El aporte de la presente investigación a las ciencias sociales de la gestión, se centra en estudiar un objeto y sujetos de estudio poco abarcados en investigaciones peruanas e internacionales, según las bases de datos de Scopus y Web of Science.

Las FinTech son startups, pues ofrecen modelos de negocio validados con alto perfil de crecimiento, combinando las finanzas con la tecnología para poder satisfacer adecuadamente las necesidades de los consumidores financieros (Tanda \& Schena, 2019). En el Perú, el sector FinTech tiene una alta mortalidad que bordea el $19 \%$ por la alta competencia (BID \& Finnovista, 2018).

Por otro lado, la gestión de la tecnología es uno de los motores del proceso de innovación (Medellín, 2010). En consecuencia, analizar los procesos de gestión tecnológica en las FinTech es esencial para poder encontrar falencias cuya mejora permita contribuir en la innovación exitosa y ayude a mejorar la competitividad de la empresa. Para analizar cualitativamente los procesos de gestión tecnológica, se utilizará el modelo de las Seis Facetas de Kearns et al. (2005) y la evaluación cualitativa de Liao et al. (2013) quienes, a partir del modelo de Kearns et al. (2005), propusieron una serie de criterios y subcriterios para evaluar cada uno de los seis procesos: evaluación, integración de producto y proceso, planeamiento, implementación, formación y cambio. Entonces, a partir de estos criterios y subcriterios, se propuso una rúbrica validada por el Grupo de Investigación de Gestión de la Innovación (GIGI) de la PUCP. Esta herramienta permitió evaluar cada proceso (variables) sobre la base de cada uno de los 32 principios que albergan (subvariables).

En este sentido, la estructura del presente estudio se propone de la siguiente manera: primero, el marco teórico, cuyas temáticas son el tema de las FinTech y la gestión de la tecnología para la innovación de servicios financieros; segundo, la metodología utilizada en las distintas fases de la investigación; y, por último, los principales resultados del estudio respecto al análisis de los procesos de gestión tecnológica de las FinTech seleccionadas.

\section{Marco Teórico}

\subsection{FinTech}

Las startups FinTech son emprendimientos tecnológicos que combinan las finanzas y la tecnología en modelos de negocios escalables (Arslanian \& Fisher, 2019). Si bien es cierto, las FinTech comenzaron a aparecer con mayor fuerza en el primer decenio del siglo XXI, según Arslanian \& Fisher (2019), hay cuatro condicionantes que han propiciado su aparición: un fluctuante escenario macroeconómico y regulatorio (con relevancia mundial desde la crisis financiera del 2008), una rápida evolución de la tecnología (con una mayor evolución anual de las TIC), crecientes expectativas de los clientes y una demanda insatisfecha y sin acceso a servicios financieros. Es importante considerar que las entidades financieras tradicionales, durante décadas, han demostrado poca capacidad para conectar con los clientes, quienes las perciben como entidades burocráticas, con altos costos en sus productos financieros y que se preocupan poco por mejorar la experiencia en el uso de sus servicios. 
En cuanto a la taxonomía del sector, los distintos emprendimientos FinTech se pueden clasificar según su actividad de intermediación financiera (Tanda \& Schena, 2019; Vodanovic Legal, 2019). De esta forma, en las soluciones de financiamiento y ahorro, se encuentran los Marketplaces, las FinTech de préstamos, crowdfunding y las soluciones de ahorro. En cuanto a los servicios y actividades de inversión, se puede mencionar las plataformas de trading, las FinTech de gestión de finanzas personales y empresariales. Entre los servicios de pago, se identifica a las FinTech de transferencias y pagos. Por último, se encuentran los servicios de seguros (InsurTech), las FinTech de puntaje crediticio y de cambio de divisas.

\subsection{Gestión de la Tecnología para la Innovación de Servicios Financieros}

La innovación es la disimilitud entre producto (bien o servicio), proceso nuevo o una combinación de estos en comparación a productos y procesos anteriores a la introducción de estos nuevos productos y procesos, incluyendo mejoras incrementales (OECD, 2018). Por otro lado, tal como lo mencionan Kanwal \& Yousaf (2019), la innovación de servicios financieros tiene una estrecha relación con el nivel de satisfacción del cliente. Por ende, estos autores proponen que las empresas de servicios financieros diseñen estrategias innovadoras, mediante el uso de TIC.

En cuanto a la gestión de la tecnología, es aquella disciplina que maximiza las ventajas competitivas, mediante el uso de tecnología y métodos organizacionales para la mejora de los procesos tecnológicos (Medellín, 2010). Entre los distintos modelos de gestión de la tecnología para empresas de rápido cambio, destaca el modelo de las Seis Facetas de Kearns et al. (2005). El modelo se compone por 6 procesos y 32 principios que, para términos de la investigación, fueron tratadas como variables y subvariables, respectivamente.

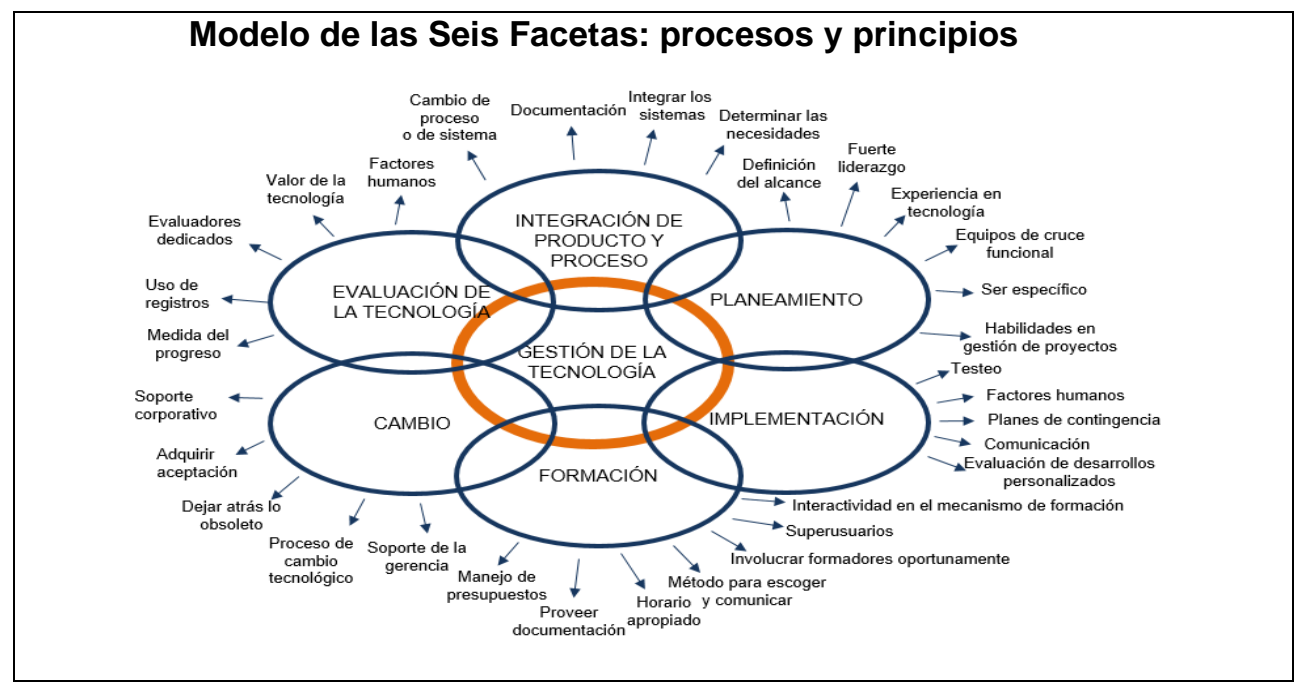

Fig. 1. Modelo de las Seis Facetas de Kearns et al. (2005). Procesos y principios.

La razón por la que se seleccionó el modelo de las Seis Facetas para analizar los procesos de gestión tecnológica de las FinTech seleccionadas, se da porque, primero, el modelo ha sido aplicado en empresas de rápido cambio tecnológico (Liao et al., 2013); segundo, porque ha sido utilizado para analizar la innovación de servicios a partir de los procesos tecnológicos (Cox, Christen, Deletic \& Fatimilehin, 2007; Liao et al., 2013; Luxmore \& Hull., 2010); y, tercero, porque ha sido aplicado en el sector financiero (Cox et al., 2007). 


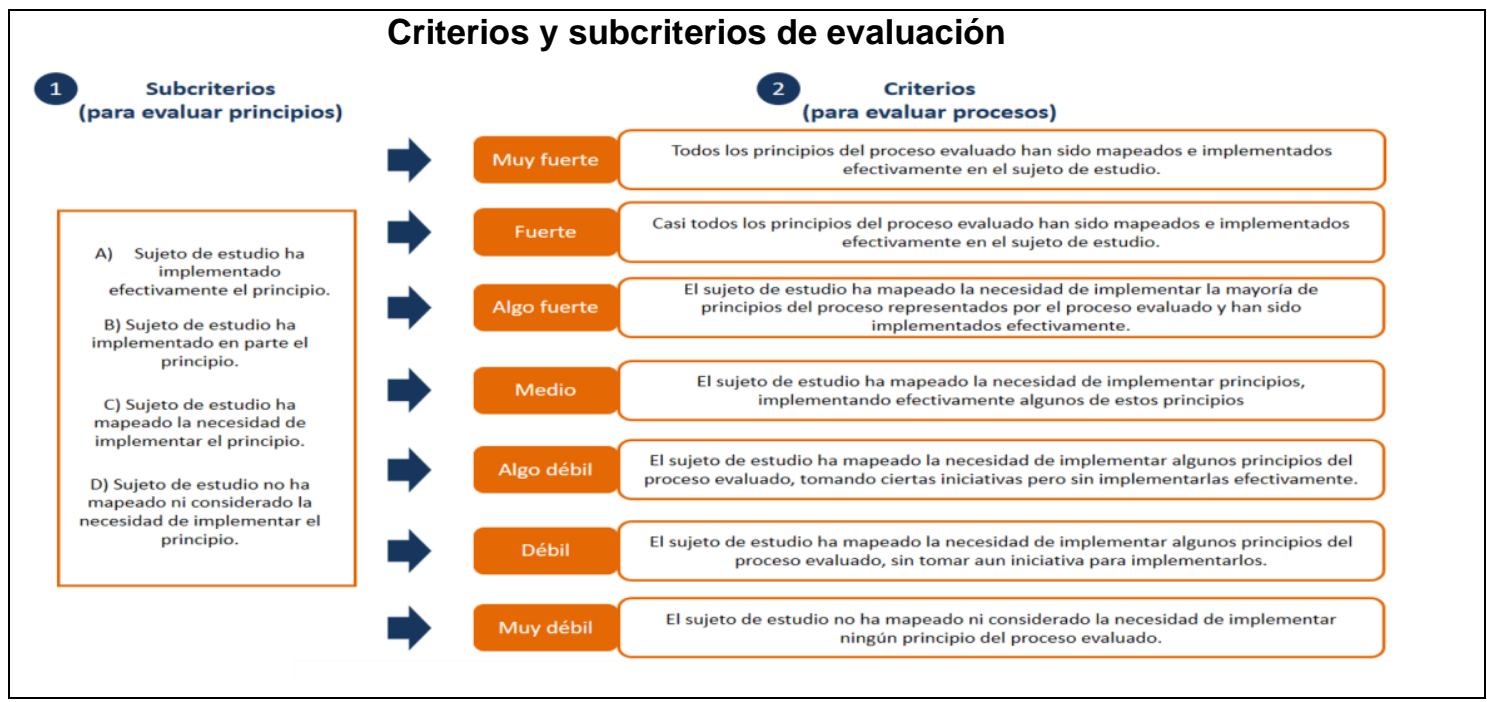

Fig. 2. Criterios y subcriterios para evaluar los procesos del modelo de las Seis Facetas según Liao et al. (2013)

Tal como se muestra en la Figura 2, el procedimiento de la evaluación de los procesos tecnológicos, utilizando el modelo de las Seis Facetas y los criterios y subcriterios de Liao et al. (2013), es el siguiente: primero, por medio de los subcriterios se evalúan los principios (subvariables) y, a partir de esto, se proceden a evaluar con los criterios los procesos tecnológicos (variables). Por último, se observa que los criterios de evaluación oscilan de muy débil a muy fuerte. Además, tal como se apreciará en la metodología, estos criterios y subcriterios permitieron construir la rúbrica de evaluación para analizar los procesos de gestión tecnológica.

\section{Metodología}

De acuerdo a la teoría metodológica de Hernández, Fernández \& Baptista (2014), la presente investigación tuvo un alcance exploratorio y descriptivo. Para poder realizar el análisis de los procesos tecnológicos de las FinTech, se revisó los anteriores estudios que aplicaron el modelo de las Seis Facetas de Kearns et al. (2005), tal como se muestra en la Tabla 1, motivo por el cual se consideró adecuado utilizar un enfoque cualitativo. Asimismo, para comprender a fondo la gestión de la tecnología, se utilizó una estrategia general de estudio de casos incrustado-múltiples (esto debido a que se estudia una unidad de análisis). De igual forma, el tipo de muestreo realizado fue no probabilístico, pues el estudio no se fundamentó en la estadística. Además, el tipo de muestreo no probabilístico fue por conveniencia. Por ende, los criterios de selección fueron determinados por el propio investigador, sobre base teórica (Hernández et al., 2014).

En consecuencia, de acuerdo a Hernández et al. (2014), se delimitaron tres criterios de selección para las FinTech: en primer lugar, tenían que pertenecer a Lima Metropolitana y encontrarse en fase de desarrollo o expansión (OECD, 2013). Esto sirvió para constatar que tuvieran sistemas formales como procesos, funciones y manejo de planes estratégicos y operativos. En segundo lugar, se delimitó que las FinTech estudiadas tengan un área de tecnología interna y que esta no sea terciarizada, lo cual aseguró que el objeto de estudio pueda analizarse dentro de los sujetos de estudio. Por último, se vio conveniente que las áreas de tecnología de las FinTech que participaron del estudio, tuvieran un CTO o gerente de tecnología y que este rol no recaiga en el CEO ni en ningún otro gerente. Sobre esto, es importante señalar que las unidades de investigación están compuestas por unidades de análisis y de observación (Hernández et al., 2014). En este sentido, el presente estudio solo tuvo una unidad de análisis que es el macro proceso de gestión de la tecnología y las unidades de observación fueron los CTO. 
Vol. 9 | Investigación Cualitativa en Ciencias Sociales: Avances y Desafíos

Tabla 1. Investigaciones que han utilizado el modelo de las Seis Facetas para evaluar los procesos de gestión tecnológica en estudio de casos

\begin{tabular}{|c|c|c|c|}
\hline Estudio & $\begin{array}{l}\text { Enfoque de la } \\
\text { investigación }\end{array}$ & Técnica utilizada & Instrumento \\
\hline Cox et al. (2007) & $\begin{array}{c}\text { Mixta (Cualitativa/ } \\
\text { Cuantitativa) }\end{array}$ & $\begin{array}{c}\text { Entrevistas/ } \\
\text { Encuesta }\end{array}$ & $\begin{array}{c}\text { Guía de } \\
\text { preguntas semi- } \\
\text { estructuradas / } \\
\text { Cuestionario de } \\
\text { encuesta }\end{array}$ \\
\hline Kearns et al. (2005) & Cualitativa & Entrevistas & $\begin{array}{c}\text { Guía de } \\
\text { preguntas semi- } \\
\text { estructuradas }\end{array}$ \\
\hline Liao et al. (2013) & Cualitativa & Análisis documental & $\begin{array}{l}\text { Registro de } \\
\text { fuentes } \\
\text { bibliográficas }\end{array}$ \\
\hline Luxmore \& Hull (2010) & Cualitativa & Análisis documental & $\begin{array}{l}\text { Registro de } \\
\text { fuentes } \\
\text { bibliográficas }\end{array}$ \\
\hline Prakhya \& Hull (2006) & Cualitativa & $\begin{array}{l}\text { Observación de } \\
\text { campo }\end{array}$ & $\begin{array}{c}\text { Guía de } \\
\text { observación de } \\
\text { campo }\end{array}$ \\
\hline
\end{tabular}

A partir de la secuencia metodológica de Yin (2003), el estudio consistió en tres fases: la fase exploratoria, la fase de recolección de información y análisis y, por último, la fase de validación de hallazgos. En primer lugar, la fase exploratoria abarcó el análisis bibliométrico y la revisión de la literatura, lo cual permitió construir una Matriz de Recolección de la Información (MRI) en la que figuran los dos principales ejes del marco teórico, con las categorías y las distintas variables de estudio extraídas a partir de la literatura. Para analizar la gestión tecnológica en las FinTech, en la MRI se operativizaron las variables y subvariables del modelo de las Seis Facetas a través de las preguntas que conformaron la guía de preguntas semiestructuradas. Es importante esclarecer que, si bien el CTO fue la unidad de observación, se complementó la información acerca del modelo de negocio de las FinTech con los aportes del CEO.

La segunda fase consistió en la recolección de la información, la posterior gestión de contenidos y el análisis de la información. Al respecto, la herramienta de recolección de información se centró en la aplicación de la guía de preguntas semiestructuradas, por medio de la técnica de entrevistas a profundidad. Por consiguiente, a los CTO se aplicaron un total de ocho entrevistas, dos entrevistas por cada CTO. En cuanto al perfil de los entrevistados, los CTO se caracterizaron por ser profesionales con conocimientos en temas de software para arquitectura on premise y en servicios de cloud computing. Asimismo, tenían más de dos años de experiencia en FinTech como gerentes del área de tecnología. De igual forma, los CEO complementaron la información acerca de las FinTech seleccionadas, en este sentido, el perfil que tuvieron es de emprendedores y fundadores de negocios FinTech que tuvieran un área de tecnología interna y con dos años o más de experiencia en dicho sector. Posteriormente, estas entrevistas se transcribieron para así procesar el contenido en el software de gestión de información cualitativa WebQDA, el cual contribuyó a brindar trazabilidad a los hallazgos.

En relación al proceso de análisis de la información, para construir los códigos árbol (tree code) y establecer el sistema de codificación en el WebQDA, se tuvo en cuenta el objetivo principal del estudio, el cual es analizar los procesos de gestión tecnológica de las FinTech. A partir de esto, previamente en la MRI, se definieron las categorías y los códigos de las variables en función del modelo de las Seis Facetas de Kearns et al. (2005). De esta forma, las variables son los seis procesos de gestión tecnológica y las subvariables codificadas fueron los 32 principios de cada proceso que propone el modelo (ver Figura 1). 
Es preciso acotar que, definir los códigos árbol contribuye a la sistematización y ordenamiento de la información, pues mediante el WebQDA se extraen las citas de las entrevistas que hacen mención a las variables y subvariables y, de esta forma, se mapean las frecuencias de cada una de las variables y subvariables de estudio en cada entrevista aplicada a los CTO. Después, se ordenaron los principales hallazgos y, a continuación, se aplicaron las dos principales herramientas de análisis utilizadas en la investigación: la rúbrica de criterios y la triangulación de perspectivas. La rúbrica (ver Figura 3) se construyó sobre los criterios y subcriterios de Liao et al. (2013) que se muestran en la Figura 2, contribuyendo en el análisis de las variables y subvariables. Por último, es oportuno mencionar que, para dotar de fiabilidad al estudio, la codificación y la sistematización de la información se realizó en dos oportunidades, de esta forma, también se contrastaron los hallazgos.

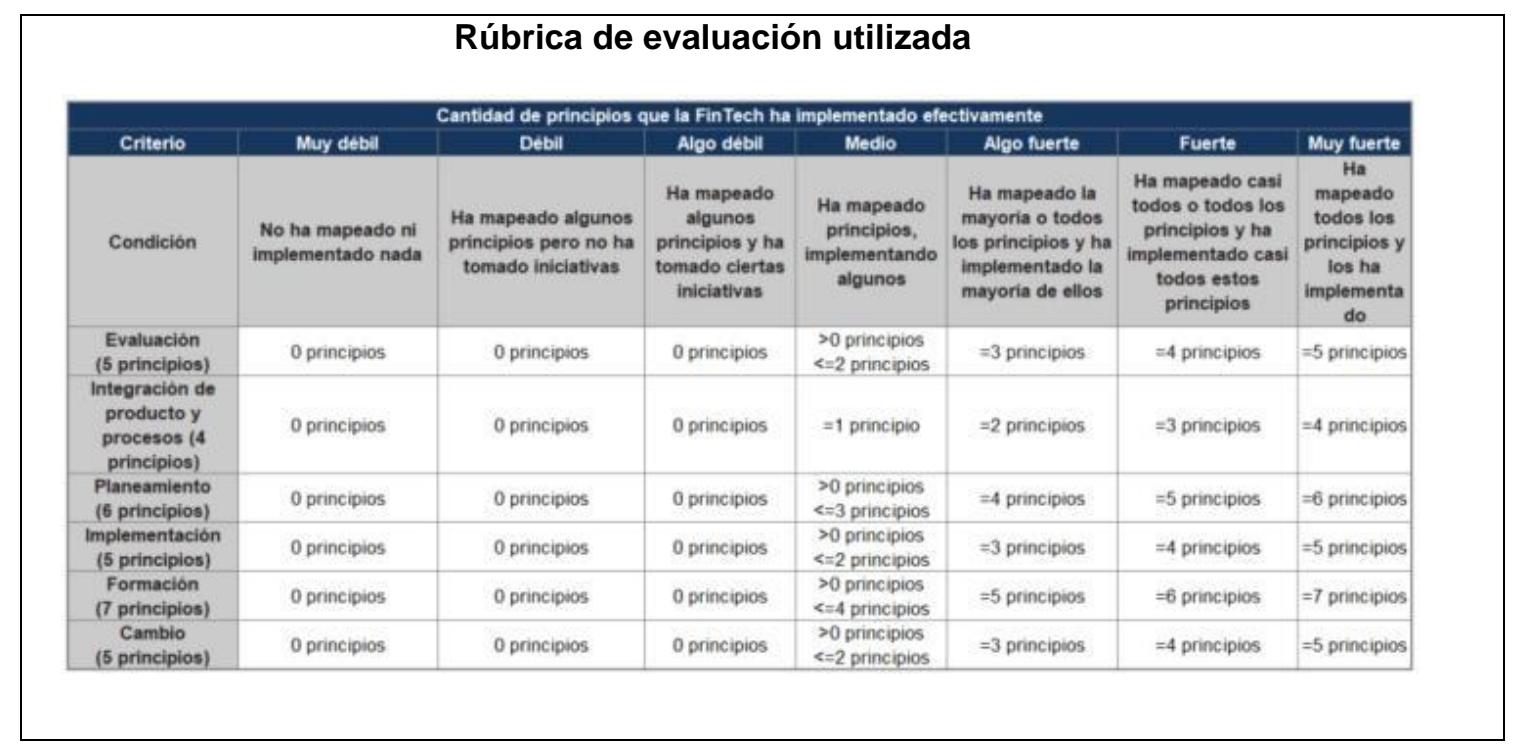

Fig. 3. Rúbrica para evaluar los procesos de gestión tecnológica de las FinTech

Finalmente, la tercera fase abarcó la validación de hallazgos obtenidos en el trabajo de campo. En esta parte, se aplicaron entrevistas a cinco expertos en temas de gestión de la tecnología, innovación y FinTech. Respecto a esto, los expertos fueron: Marco Gusukuma, profesor de la PUCP en la facultad de ingeniería experto en temas de gestión tecnológica e innovación; Amparo Nalvarte, emprendedora en FinTech con larga trayectoria y fundadora del primer neobanco peruano (B89); Erick Rodríguez, actual directivo de la Asociación FinTech del Perú con conocimiento del sector FinTech y de la parte regulatoria; Luis Gonzaga, Director Strategic Value Banking en Everis y experto en temas FinTech y, por último, el profesor Carlos Hernández, un experto cuya opinión también resultó muy valiosa pues es actual Director de la Maestría en Gestión y Política de la Innovación y la Tecnología de la PUCP y CEO de la FinTech Agente Cash. Además, también fue CTO de esta FinTech.

De forma similar a las entrevistas aplicadas a los CTO, las entrevistas a expertos se transcribieron y se utilizó el WebQDA para sistematizar la información. Además, mediante la herramienta de análisis de la triangulación de perspectivas, se compararon los hallazgos obtenidos a partir de las entrevistas a los CTO con las entrevistas aplicadas a los expertos. Finalmente, es muy importante mencionar la ética de la presente investigación, el cual se basó, en primer término, en el respeto a las distintas fuentes bibliográficas y, en segundo término, se utilizaron consentimientos informados donde cada entrevistado brindó su conformidad para el uso de la información hacia fines académicos. 


\section{Resultados}

Después de gestionar el contenido con el WebQDA y tras aplicar la rúbrica cualitativa y la triangulación de perspectivas con expertos, se dividieron los resultados de acuerdo a cada una de las seis variables de estudio (procesos).

En primer lugar, en cuanto a la variable de la evaluación de la tecnología, las dos principales subvariables que han sido implementadas efectivamente en las FinTech estudiadas son los evaluadores dedicados (todas las FinTech tienen personal de tecnología que se encargan de evaluar qué tecnologías requieren para implementarlas en sus operaciones) y el valor de la tecnología (forma en que la tecnología contribuye en el modelo de negocio). Las subvariables que más adolecen las FinTech estudiadas son la medición del progreso (en el cual resalta solo TasaTop, pues miden el progreso usando métricas de tiempo, uso de recursos humanos y financieros) y uso de registros (destaca Difondy, dado que agendan reuniones con otros CEO y especialistas para poder conocer y optar apropiadamente por una nueva tecnología). Por último, en cuanto a la subvariable factores humanos, que se relaciona a la amigabilidad e interactividad del canal por donde se imparten los servicios, figuran Apurata y TasaTop por el user experience en sus plataformas web. Tras aplicar la rúbrica de criterios y subcriterios, esta variable muestra a TasaTop (Fuerte), Apurata y Difondy (Algo fuerte) y Tranzfer.me (Débil).

En segundo lugar, en cuanto a la variable de integración de producto y proceso destacan las subvariables cambio de proceso o de sistema (según lo validado por expertos, el principal cambio es la automatización) y la subvariable de determinar las necesidades (las FinTech estudiadas conciben bien qué problemas tienen en sus áreas de tecnología), siendo aplicadas en todas las FinTech. Por su parte, la subvariable de integración de sistemas, es aplicada por Apurata y TasaTop (manejan procesos integrados). Por último, una subvariable poco aplicada en las FinTech es la documentación, que puede ahorrar costos, pues al trabajar con lenguajes de programación, es necesario documentar estos códigos (solo Apurata lo aplica). En cuanto al criterio de evaluación cualitativa que cumplen las FinTech, figura Apurata (Muy fuerte), TasaTop (Fuerte) y Difondy y Tranzfer.me (Algo fuerte).

En tercer lugar, en cuanto a la variable planeamiento, destacan todas las FinTech estudiadas, considerando que han implementado efectivamente subvariables tales como fuerte liderazgo, experiencia en tecnología y ser específico. Estas subvariables se relacionan con las habilidades blandas del CTO y con el expertise de todo el equipo de tecnología (las FinTech estudiadas cuentan con estos requisitos). Asimismo, la mayoría de las FinTech ha implementado equipos de cruce funcional (a excepción de Tranzfer.me, las FinTech comunican a sus equipos cómo va el proyecto de implementación de nueva tecnología). Respecto a la subvariable definición del alcance, resaltan Apurata y TasaTop, pues planean en términos de recursos humanos, financieros y tecnológicos. Por último, en cuanto a la subvariable habilidades en gestión de proyectos, figuran Difondy y Tranzfer.me, dado que los CTO no solo han aprendido a gestionar proyectos por experiencia, sino que se han certificado en temas relacionados a proyectos con metodologías ágiles. En relación al criterio de evaluación que cumplen las FinTech, destacan Apurata, Difondy y TasaTop (Fuerte), mientras que Tranzfer.me cumple con un criterio "Algo fuerte".

En cuarto lugar, en la variable de implementación tecnológica, sobresale la subvariable de testeo, puesto que las FinTech estresan las nuevas tecnologías o desarrollos que implementan. Asimismo, la mayoría de las FinTech (a excepción de Difondy) cumple con la subvariable factores humanos (mapean cómo la nueva tecnología va a impactar en la dinámica de trabajo). En cuanto a la subvariable comunicación, los expertos señalan la importancia de ser efectivo informando avances. En este sentido, destacan Difondy y TasaTop. Respecto a la subvariable planes de contingencia, figura Apurata, pues utilizan herramientas de monitoreo que les da capacidad de reacción frente a fallos. Por último, en cuanto a la subvariable evaluación de desarrollos personalizados, no se encontró en el trabajo de campo (tal como señalan los expertos, por temas de recursos esta subvariable se aplica especialmente en empresas grandes). 
Respecto al criterio de evaluación que cumplen, resaltan Apurata y TasaTop (Fuerte) seguidos por Difondy y Tranzfer.me (Algo fuerte).

En quinto lugar, la formación tecnológica es la variable en la que más adolecen las FinTech estudiadas debido a que no aplican una adecuada formación a los clientes para que puedan utilizar los servicios financieros que ofertan. Sobre la subvariable manejo de presupuestos, únicamente Tranzfer.me destaca, dado que destinan dinero para enseñar a sus clientes a utilizar sus servicios. Respecto a la subvariable proveer documentación, todas las FinTech a excepción de Tranzfer.me tienen manuales o muestran procedimientos en sus plataformas que el cliente debe seguir para demandar sus servicios. En relación a la subvariable horario apropiado, ha sido implementada por Difondy y Tranzfer.me, pues aplican la formación a clientes antes de lanzar un nuevo servicio con nuevas implementaciones. Apurata y TasaTop destacan en las subvariables método para escoger y comunicar e interactividad del mecanismo de formación, relacionadas en torno al canal por donde la FinTech decide formar a sus clientes para el uso de sus servicios. En cuanto a la subvariable involucrar formadores oportunamente, todas las FinTech cumplen a cabalidad este principio, puesto que ayudan a atender dudas de clientes acerca del uso de los servicios y, por último, respecto a la subvariable superusuarios, TasaTop y Tranzfer.me presentan este principio (ambas obtienen retroalimentación de los clientes). Respecto a esto, los expertos señalan que la retroalimentación de los clientes contribuye en la mejora del servicio. En relación al criterio de evaluación que cumplen, figura TasaTop (Algo fuerte), mientras que las demás FinTech cumplen con un criterio Medio.

En sexto lugar, sobre la variable cambio tecnológico resaltan las subvariables dejar atrás lo obsoleto (desactivar tecnología que genere costos a la FinTech) y proceso de cambio tecnológico (tener en cuenta de qué forma la nueva tecnología implementada cambia los procesos), siendo ambas implementadas efectivamente en todas las FinTech estudiadas. En cuanto a la subvariable soporte corporativo, TasaTop es la única que aplica este principio (maneja un plan de gestión de talento para el personal del área de tecnología). Respecto a la subvariable adquirir aceptación, la mayoría de las FinTech, excepto Apurata, comunica efectivamente a los clientes los principales cambios en el servicio tras nuevas implementaciones. Por último, en cuanto a la subvariable soporte de la gerencia, destaca TasaTop, pues la gerencia brinda apoyo y soporte al equipo en el proceso de cambio. Tras la aplicación de la rúbrica de criterios, resaltan TasaTop (Muy Fuerte), seguidos por Difondy y Tranzfer.me (Algo fuerte) y Apurata (Medio).

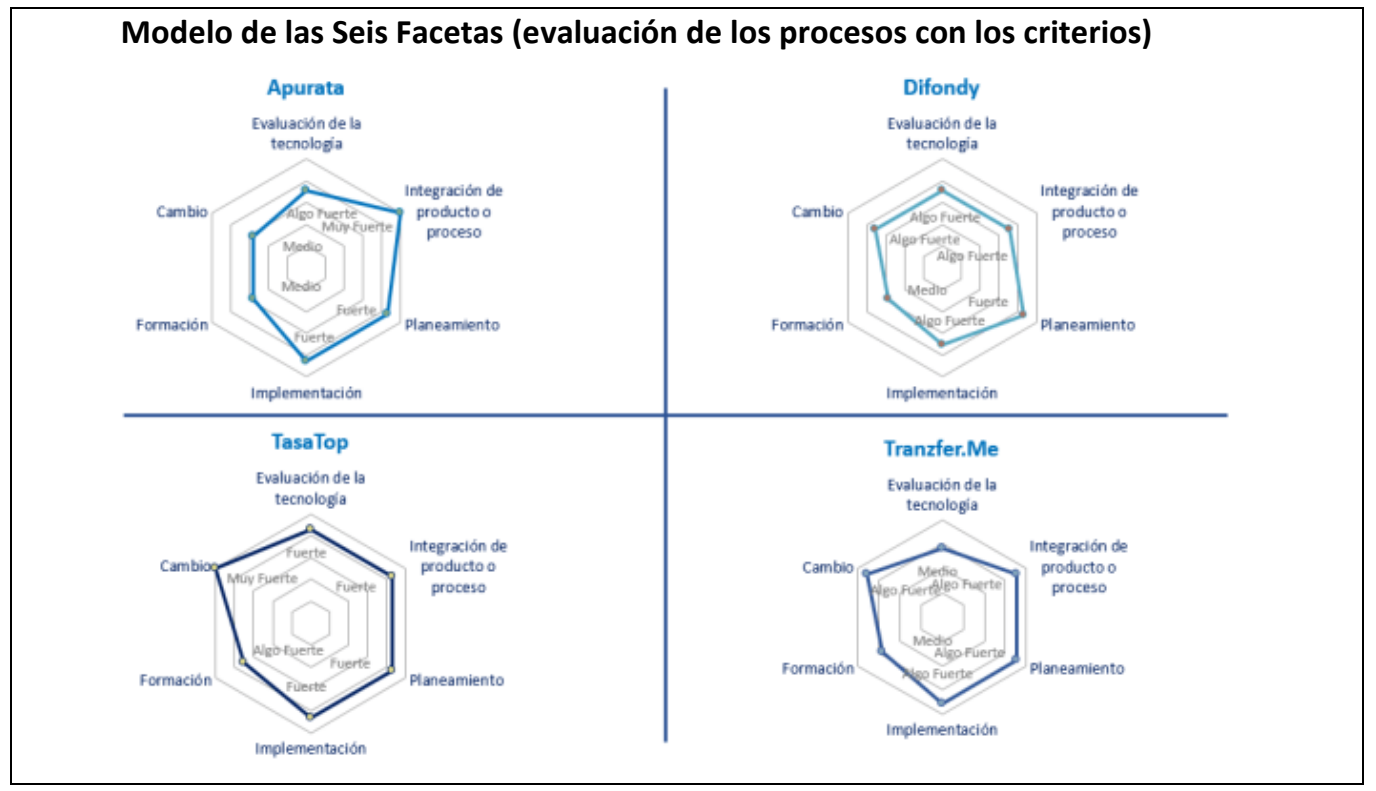

Fig. 4. Evaluación de los procesos tecnológicos de las FinTech estudiadas 


\section{Conclusiones}

Como se aprecia, la metodología cualitativa utilizada en la presente investigación, permitió cumplir con el objetivo principal de analizar los procesos de gestión tecnológica de las FinTech analizadas mediante el modelo de las Seis Facetas. Además, tal como señalan los expertos, evaluar la gestión tecnológica en FinTech puede resultar un problema para los gerentes de tecnología (motivo por el cual no lo priorizan). Al respecto, si bien el problema de investigación es la falta de un análisis de los procesos de gestión de la tecnología de las FinTech seleccionadas, se denota que una forma viable de analizar los procesos de gestión tecnológica en estas FinTech, es aplicando técnicas cualitativas. De igual modo, se observa que las entrevistas a profundidad por medio de guía de preguntas semiestructuradas, posibilitaron encontrar información enriquecedora respecto a las variables y subvariables de estudio, permitiendo estudiar ampliamente la unidad de análisis en los sujetos de estudio. De esta forma, se concluye que se puede recurrir al instrumento de guía de preguntas semiestructuradas para poder obtener información útil acerca de cómo se va gestionando los procesos de gestión tecnológica en FinTech.

Por otro lado, si bien es cierto, por la naturaleza del estudio no se pueden generalizar las conclusiones, se evidencia que el uso del software WebQDA fue útil para sistematizar la información y la aplicación de las distintas herramientas de análisis cualitativo. Por consiguiente, los resultados permiten determinar que las FinTech estudiadas cuentan con procesos tecnológicos formales (identifican que cuentan con determinados procesos) y con procesos tecnológicos no formales (no identifican que cuentan con determinados procesos, aunque lo aplican). Asimismo, la principal contribución a las metodologías cualitativas es proponer al modelo de las Seis Facetas como una herramienta para evaluar cualitativamente los procesos de gestión tecnológica de FinTech que tengan un área de tecnología interna.

Esto se materializó con la aplicación de la rúbrica diseñada para este fin, lo cual permitió concluir que el planeamiento es el proceso más fuerte en las FinTech estudiadas, pues cumplen con un criterio mayor o igual a "Algo fuerte" (debido a que han implementado efectivamente tres principios: fuerte liderazgo, experiencia en tecnología y ser específico). Por otra parte, el proceso de formación representa un reto en todas las FinTech, dado que en este proceso la mayoría cumple con un criterio "Medio". Únicamente TasaTop cumple con un criterio "Algo fuerte" debido a que ha implementado algunos mecanismos para comunicar su propuesta de valor, enseñando a los usuarios cómo utilizar los distintos servicios que proveen.

Finalmente, tras el análisis de las 6 subvariables (procesos) y las 32 subvariables (principios) del modelo, TasaTop se erige como la FinTech más preparada para innovar en sus servicios. Esto se refleja en que no son una FinTech monoproducto, sino que brindan distintos productos de ahorro e inversión, convirtiéndose en una FinTech multiproducto (Marketplace). Es imperativo mencionar que, llegar a consolidarse como una FinTech Marketplace representa, para los expertos consultados, el futuro de las FinTech.

\section{Agradecimientos}

Un agradecimiento especial al Grupo de Investigación de Gestión de la Innovación (GIGI) de la PUCP por el fructífero apoyo brindado.

\section{Referencias}

Arslanian, H., \& Fischer, F. (2019). The Future of Finance: The Impact of FinTech, Al, and Crypto on Financial Services. Switzerland: Springer Nature.

BID, \& Finnovista. (2018). FINTECH: América Latina 2018 | Crecimiento y consolidación. Recuperado de https://publications.iadb.org/publications/spanish/document/Fintech Am\%C3\%A9rica-Latina-2018-Crecimiento-y-consolidaci\%C3\%B3n.pdf 
Cox, S., Christen, M., Deletic, G., \& Fatimilehin, O. (2007). Technology management analysis for effective implementation of change using the six facets model: evidence from within a financial services company. Journal of International Business Strategy, 7(1), 33-44.

Hernández, R., Fernández, C., \& Baptista, P. (2014). Metodología de la investigación (5.a ed.). McGraw-Hill Education.

Kanwal, R., \& Yousaf, S. (2019). Impact of Service Innovation on Customer Satisfaction: An Evidence from Pakistani Banking Industry. Emerging Economy Studies, 5(2), 125-140. Recuperado de https://doi.org/10.1177/0976747919870876

Kearns, M., Taylor, J., \& Hull, C. (2005). The six facets model: Technology management in the effective implementation of change. International Journal of Innovation and Technology Management (IJITM), 2, 77-100. Recuperado de https://doi.org/10.1142/S0219877005000381

Liao, A., Hull, C., \& Sriramachandramurthy, R. (2013). The six facets model of technology management: A study in the digital business industry. International Journal of Innovation and Technology Management, 10(4), 1350019-1. Recuperado de https://doi.org/10.1142/S0219877013500193

Luxmore, S., \& Hull, C. (2010). Externalities and the six facets model of technology management: Genetically modified organisms in agribusiness. International Journal of Innovation and Technology Management (IJITM), 7, 19-36. Recuperado de https://doi.org/10.1142/S0219877010001799

Medellín, E. (2010). Gestión tecnológica en empresas innovadoras mexicanas. Review of Administration and Innovation, 7(3), 58-78. Recuperado de https://doi.org/10.5585/RAI.2010684

OECD (2013). Startup América Latina: Promoviendo la innovación en la región, Estudios del Centro de Desarrollo, OECD Publishing. Recuperado de http://dx.doi.org/10.1787/9789264202320-es

OECD. (2018). Oslo Manual 2018-Guidelines for Collecting, Reporting and Using Data on Innovation (4 $4^{\underline{a}}$ ed). Paris: OECD Publishing. Recuperado de http://www.oecd.org/science/oslomanual-2018-9789264304604-en.htm

Tanda, A., \& Schena, C.M. (2019). FinTech, BigTech and Banks: Digitalisation and its Impact on Banking Business Models (1a ed.). Switzerland: Springer Nature. Recuperado de https://10.1007/978-3-030-22426-4

Vodanovic Legal (2019). Panorama de la Industria Fintech en Perú. Recuperado de https://vodanovic.pe/panorama-de-la-industria-fintech-en-peru-actualizado-junio-2019/

Yin, R. (2003). Case study research: Design and Methods. Thousand Oaks, Calif: Sage Publications.Teixeira-Dias, J. J. C., Pedrosa de Jesus, H., Neri de Souza, F., \& Watts, D. M. (2005). Teaching for Quality Learning in Chemistry. International Journal of Science Education, 27(9), 1123-1137. 